\title{
Efficacy and safety of iota-carrageenan nasal spray versus placebo in early treatment of the common cold in adults: the ICICC trial
}

\author{
R. Eccles ${ }^{1}$, B. Winther ${ }^{2}$, S.L. Johnston ${ }^{3}$, P. Robinson ${ }^{4}$, M. Trampisch ${ }^{5}$ and S. Koelsch ${ }^{6 *}$
}

\begin{abstract}
lota-carrageenan (I-C) is active against respiratory viruses in vitro and was effective as nasal spray in three previous clinical trials. The current trial served to further investigate I-C in patients with early common cold symptoms.

Methods: This randomized, placebo-controlled, double-blind phase IV trial was conducted in 200 adult patients with self-diagnosed colds of $<48 \mathrm{~h}$ ' duration that were confirmed by baseline cold symptom scores. Patients were to self-administer $0.12 \% \mathrm{I}-\mathrm{C}$ or placebo spray ( $\mathrm{NaCl} 0.5 \%)$ four times daily for four to ten days and record symptom information for ten days. Common respiratory viruses were quantified by RT-PCR during pretreatment and on Day 3 or 4. The primary endpoint was the mean total symptom score (TSS) of eight cold symptoms on Days 2-4 (TSS $2_{-4}$ ).

Results: Patients in both treatment groups had similar baseline TSSs (mean TSS: 6.75 for I-C and 6.79 for placebo). Viruses were detected in baseline samples from 53 of $98 \mathrm{I}-\mathrm{C}$ patients $(54.1 \%)$ and 54 of 97 placebo patients (55.7 \%). Mean \pm SE for $\mathrm{TSS}_{2-4}$ was $5.78 \pm 0.25$ for $\mathrm{I}-\mathrm{C}$ patients and $6.39 \pm 0.25$ for placebo $(p=0.0895)$. Exploratory analyses after unblinding (TSS ${ }_{2-4}$ excluding a patient with aberrantly high symptom scores $\left[\mathrm{TSS}_{2-4}\right.$, ex 1pt]; mean of TSS over Days 1-4 $\left[\mathrm{TSS}_{1-4}\right]_{\text {; }}$ change in $\mathrm{TSS}_{1-4}$ relative to baseline $\left[\mathrm{TSS}_{1-4}\right.$, rel]) demonstrated treatment differences in favor of I-C ( $p=0.0364, p=0.0495$ and $p=0.0421$, respectively). For patients with quantifiable rhinovirus/enterovirus at baseline, there was a trend towards greater reduction of virus load at Day 3 or 4 ( $p=0.0958$; I-C: $90.2 \%$ reduction in viral load; placebo: $72.0 \%$ ). Treatments were well tolerated with no differences in adverse event rates.
\end{abstract}

Conclusions: The primary endpoint did not demonstrate a statistically significant difference between I-C and placebo but showed a trend towards I-C benefit. Exploratory analyses indicated significant reduction of cold symptoms in the I-C group relative to placebo during the first four days when symptoms were most severe, and also substantiated I-C's activity against rhinovirus/enterovirus.

Trial registration: NCT01944631 (clinicaltrials.gov)

Keywords: lota-carrageenan, Common cold, Human rhinovirus, Clinical trial

\section{Background}

The common cold is caused by a variety of respiratory viruses, such as human rhinoviruses (HRV), coronaviruses, human enteroviruses (HEV), respiratory syncytial virus (RSV), parainfluenza viruses, or influenza viruses [1]. Rhinoviruses are the most common cause of respiratory tract infections in individuals of all ages. In adults, rhinoviruses cause approximately $50 \%$ of common colds and up to $90 \%$ of colds during the autumn epidemic season.

\footnotetext{
* Correspondence: stephan.koelsch@boehringer-ingelheim.com ${ }^{6}$ Boehringer Ingelheim Pharma GmbH \& Co. KG, CHC Development, Medicine \& Regulatory Affairs, Ingelheim/Rhein 55216, Germany Full list of author information is available at the end of the article
}

Common colds are frequent illnesses in both children and adults; on average, adults report 2.5 episodes per year [2]. It has been estimated that the total economic impact of non-influenza-related viral upper respiratory tract infections approaches $\$ 40$ billion annually [2], and such infections can result in serious and even life-threatening sequelae in patients with underlying illnesses such as asthma, COPD or immune compromise. With the exception of influenza and RSV, there are no vaccinations or anti-viral medicinal products available for treatment of infection with the viruses that cause the common cold.

Iota-carrageenan (I-C) - a sulfated polysaccharide found in some species of red seaweed (Chondrus crispus) - has 
demonstrated antiviral activity against respiratory viruses in cell culture and in animal models [3, 4]. The I-C polymer seems to bind directly to viruses, preventing viral attachment to host cells. In vitro and in vivo studies have demonstrated the effectiveness of I-C against several viruses such as HRV [4] and influenza A [3]. In vitro tests have established that I-C does not penetrate freshly excised bovine nasal mucosa, and therefore is not absorbed systemically (data on file, Marinomed Biotechnology $\mathrm{GmbH}$ ). Carrageenan is generally recognized as safe (GRAS) for use in food and topical applications. Because the primary site of infection and replication of most cold-causing viruses is the nasal mucosa, it was speculated that early and targeted treatment of the nasal mucosa with I-C may block viral entry at the level of the respiratory mucosa, and interfere locally with the propagation of viral replication.

Therefore, a nasal spray containing $0.5 \%$ saline and $0.12 \%$ iota-carrageenan (I-C nasal spray) has been developed and registered as a medical device. This product has recently been licensed to Boehringer Ingelheim, the sponsor of the current study. Between 2008 and 2011, three randomized clinical trials (two in adults and one in children) were conducted comparing I-C nasal spray with saline solution (placebo). In all 3 trials, there were indications of efficacy, including significantly reduced cold symptoms [5]; positive effects on symptoms in patients in whom less co-medication or no co-medication was used [6]; significantly reduced viral loads [5-7]; and faster reduction of common cold symptoms [6, 7]. Treatments were safe and well tolerated [5-7].

The ICICC trial (ICICC: Iota-Carrageenan In Common Cold) was designed as a controlled evaluation of the safety and efficacy of I-C in the treatment of patients with early common cold symptoms. The effects of treatment on cold symptoms and the duration of the cold and on viral load were assessed.

\section{Methods}

\section{Trial design and patient population}

The ICICC trial was conducted at the Common Cold Center, Cardiff, Wales, UK. It was a randomized, placebocontrolled, double-blind, two-arm parallel group trial, with the aim of investigating the efficacy of I-C nasal spray in comparison with placebo nasal spray for the treatment of early common cold in adults ( $\geq 18$ years). The trial was registered under the clinicaltrials.gov accession number NCT01944631.

Volunteer patients were recruited by poster advertisements around the Cardiff University campus and by e-mail to the students and staff of Cardiff University. Based on the results of a previous trial [5], it was calculated that a sample size of 93 in each group would have $90 \%$ power to detect the expected difference in means between the $\mathrm{TSS}_{2-4}$ scores of the groups (see below for definition of $\mathrm{TSS}_{2-4}$ ); it was therefore planned to include a total of 200 patients eligible for randomization. To be eligible for participation, patients were to have had common cold symptoms for $\leq 48$ h before trial entry, based on self-reporting during the screening interviews. Patients were to rate eight common cold symptoms (headache, muscle ache, chilliness, sore throat, blocked nose, runny nose, cough, and sneezing) on a 0 to 3 rating scale [8] $(0=$ no symptoms, $3=$ maximum severity $)$ and were to have a total symptom score (the sum of these eight common cold symptoms at baseline) of $\leq 9$. Patients were also to have a score of $\geq 1$ for at least one of the following symptoms: sore throat, runny nose, or blocked nose which reflects the standard cold study design according to Jackson.

Inclusion criteria constructed similarly to the previous 3 clinical trials conducted with I-C. Patients with high symptom scores $(\geq 9)$ were excluded in order to recruit only patients in the early stages of a cold and exclude subjects with later infections. This strategy was designed to start treatment early to optimize the chance to improve the clinical course of a cold. This early intervention strategy may have been partially responsible for lowering the power of the study, since the proportion of patients in the trial who did not develop full-blown colds was relatively high (reflected by only about $24 \%$ rhinovirus-positive patients; see Results and Discussion).

\section{Trial treatment and outcome measures}

Patients were instructed to self-administer 1 puff $(0.14 \mathrm{~mL})$ of trial medication to each nostril 4 times per day. Trial medication was either I-C nasal spray $(1.20 \mathrm{~g}$ iotacarrageenan $/ \mathrm{L}$ in $0.5 \% \mathrm{NaCl})$ or placebo $(0.5 \% \mathrm{NaCl})$. Both patients and investigators were blinded to treatment allocation. Treatment was to be mandatory for 4 days, and depending on patient preference, could be continued for up to 6 additional days, resulting in a maximum treatment duration of 10 days.

Patients were to record their symptoms once per day in the evening of Days 1-10 in a symptom diary, using the $0-3$ rating scale for each of the eight cold symptoms (see above). The primary endpoint of the trial was the mean total symptom score (TSS) of those documented eight single cold symptoms calculated as the averaged daily sum over Days $2-4\left(\mathrm{TSS}_{2-4}\right)$. Secondary endpoints included the mean of the daily sum of three systemic common cold symptoms (headache, muscle ache, and chilliness) over Days 2-4 ( $\left.\mathrm{SSS}_{2-4}\right)$; the mean of the daily sum of 5 single local common cold symptoms (sore throat, blocked nose, runny nose, cough, and sneezing) over Days 2-4 ( $\left.\mathrm{LSS}_{2-4}\right)$; area under the curve (AUC) of daily symptom scores over the 10-day period (AUC-TSS ${ }_{1-10}$ ) calculated as the sum of the eight single cold symptoms over 
Days 1-10; duration of the cold (as determined by patients' answer in the diary to the question "Do you still have a cold?" at the end of each treatment day); and the patients' assessment of efficacy as evaluated at the end of trial visit, at which patients answered the question "How effective was the treatment in relieving your common cold symptoms?" using a $0-4$ scale $(0=$ poor, $1=$ fair, 2 = good, $3=$ very good, or 4 =excellent). Further pre-specified endpoints included TSS at each study day (Days 1-10) and viral load (change from baseline) on Day 3 or 4 for various virus types. After trial data were unblinded, three exploratory analyses were performed: assessment of $\mathrm{TSS}_{2-4}$ excluding a patient with aberrantly high symptom scores $\left(\mathrm{TSS}_{2-4}\right.$, ex $\left.1 \mathrm{pt}\right)$; the mean of the TSS over Days 1-4 ( $\left.\mathrm{TSS}_{1-4}\right)$; and change in $\mathrm{TSS}_{1-4}$ relative to baseline $\left(\mathrm{TSS}_{1-4}\right.$, rel) to adjust for the potential impact of baseline TSS $\left(\mathrm{TSS}_{0}\right)$ on $\mathrm{TSS}_{1-4}\left(\mathrm{TSS}_{0}\right.$ scores ranged from 3.00-9.00 in the I-C group and from 2.00-9.00 in the placebo group). To calculate $\mathrm{TSS}_{1-4}$, rel, $\mathrm{TSS}_{0}$ was subtracted from $\mathrm{TSS}_{1-4}$ and the result was divided by $\mathrm{TSS}_{0}$. In addition, $\mathrm{TSS}_{2-4}$ scores were calculated for the virus positive subset and the $\mathrm{HRV} / \mathrm{HEV}$ positive subset (see Analysis sets below for definition). Safety was assessed on the basis of incidence of treatmentemergent adverse events (AEs) and overall tolerability was assessed by the patients themselves and recorded by the investigator at the final visit.

\section{Viral assessment}

Respiratory viruses and viral load were determined from nasopharyngeal lavage samples obtained at baseline and on either Day 3 or 4 [9]. Viral presence was to be determined for all patients using both qualitative and quantitative tests for respiratory viruses. Real-time polymerase chain reaction (RT-PCR) was used for qualitative determinations of the presence of influenza $\mathrm{A}$ and $\mathrm{B}$, picornaviruses, $\mathrm{HRV} / \mathrm{HEV}$, human bocavirus, human metapneumovirus, coronaviruses 229E, HKU1, NL63, and OC43, adenovirus, human parechovirus, respiratory syncytial virus, and parainfluenza virus types 1-4. Quantitative viral loads for HRV and HEV were determined using the panenterhino/Ge/08 assay $[10,11]$. Quantitative viral loads for influenza A and B and coronaviruses 229E, HKU1, NL63, and OC43 were assessed according to Garbino et al. [12, 13]. The panenterhino/Ge/ 08 assay provided quantifiable HRV and HEV viral load results; however, in some patients, virus was detectable but viral loads were below the level of quantification of the test. Such patients were considered to be virus-positive, but non-quantifiable (that is, a qualitative positive result). The total number of virus-positive patients was obtained by summing up the number of patients who had a positive result on any qualitative assay, or who had a detectable quantitative (quantifiable or non-quantifiable) result on the panenterhino/Ge/08 assay.

\section{Analysis sets}

Two main analysis sets (the treated set and the full analysis set [FAS]) were evaluated. The treated set included all patients who were documented to have taken at least one dose of trial medication, and was used for safety analyses. The FAS included patients documented to have taken at least one dose of trial medication, for whom a baseline TSS was recorded, and who supplied data for assessment of the primary endpoint. The FAS was used for analysis of the primary and secondary endpoints. Three analysis subsets within the FAS were also defined after unblinding, and included 1) the virus positive subset (those patients whose nasal lavage specimens yielded a least one positive qualitative or a detectable quantitative [quantifiable or non-quantifiable] result at baseline); 2) the HRV/HEV positive subset (those patients whose lavage yielded a least a detectable [quantifiable or nonquantifiable] result on the panenterhino/Ge/08 assay at baseline); and 3) the quantifiable HRV/HEV subset (those patients who had a quantifiable result on the panenterhino/Ge/08 assay at baseline). These subsets were used for analysis of additional exploratory endpoints.

\section{Statistical methods}

The primary endpoint $\mathrm{TSS}_{2-4}$ was analyzed using an analysis of covariance (ANCOVA) adjusting for continuous covariates ' $\mathrm{TSS}_{0}$ ' and 'treatment'. Differences between I-C and placebo treatment groups were assessed based on the adjusted means and the corresponding two-sided $95 \%$ confidence intervals (CIs). The secondary endpoints $\mathrm{SSS}_{2-4}$, $\mathrm{LSS}_{2-4}$, and AUC-TSS $1-10$ (adjusted for $\mathrm{TSS}_{0}$ and treatment) were analyzed similarly. The three exploratory endpoints $\mathrm{TSS}_{2-4 \text {, ex 1pt }}, \mathrm{TSS}_{1-4}$, and $\mathrm{TSS}_{1-4}$, rel were analyzed in the same way as the primary endpoint, that is, by adjusting for ' $\mathrm{TSS}_{0}$ ' and 'treatment'. For all the above endpoints, missing single symptom scores at baseline and at any post-baseline measurement time were replaced by zero, if at least one of the eight symptom scores was available. Thereafter, missing daily TSS, LSS and SSS data were replaced the last observation carried forward procedure.

The duration of the cold was examined by recording the time to loss of cold, defined by the first "no" response to the question "Do you still have a cold?" in the patient's daily symptom diary. The log rank test stratifying for $\mathrm{TSS}_{0}$ was used to compare treatment groups. The endpoint variable was censored at day 10 if a patient did not recover from his or her cold. To test whether patients' assessment of efficacy differed between the I-C group and the placebo group, an ordinal logistic regression model adjusting for $\mathrm{TSS}_{0}$ was applied to the efficacy score $(0-4)$ provided by patients. Missing entries were assigned the least favorable category. 
The analysis of the endpoint variable TSS at each study day (Days 1-10) used a restricted maximum likelihood-based repeated measures approach, using all longitudinal TSS data from Days 1-10. The statistical model included the fixed categorical effects of treatment, day and treatment-by-day interaction and the continuous covariate of $\mathrm{TSS}_{0}$. An unstructured covariance structure was used to model within-patient errors. Missing single symptom scores at baseline and at any postbaseline measurement time were replaced by 0 , if at least one of the 8 symptom scores was available. If a patient had a missing TSS at a specific day and all subsequent days until Day 10 and the cold ended on that day (that is, the question "Do you still have a cold" was answered by "no" at that day at latest), the missing TSS values for that and the subsequent days until Day 10 were replaced by 0 . For all other cases no imputation of missing TSS values was performed.

The mean change in viral load from baseline was adjusted for the continuous covariates 'baseline viral load' and 'treatment' using an ANCOVA. Differences between I-C and placebo treatment were assessed based on the adjusted means and the corresponding $95 \%$ CIs, which were calculated using a two-sided approach. For quantitative viral load analyses, missing values and values below the limit of quantification (LOQ) were replaced with the LOQ value.

\section{Results}

Approximately 800 patients were evaluated with respect to symptomatology and duration of symptoms (prescreening); of these, 201 were formally enrolled and screened. Of the enrolled patients, one was not randomized, and 200 were randomized and treated (I-C, $N=$ 100 ; placebo, $N=100)$. Overall, $97.0 \%$ of the 200 entered patients completed trial treatment, and $3.0 \%$ prematurely discontinued treatment with trial medication. The proportion of patients discontinuing treatment was similar for the treatment groups (I-C: 2 of 100 patients, $2.0 \%$; placebo: 4 of 100 patients, $4.0 \%$ ). The most frequent reason for trial discontinuation was being lost to follow-up (I-C: 1 patient; placebo: 3 patients). Of the 200 treated patients, 98 in the I-C group and 97 in the placebo group provided data for the primary endpoint and were included in the FAS (Fig. 1).

Including the 200 patients who were treated, baseline demographic characteristics were comparable between the I-C and placebo groups (Table 1).

\section{Primary endpoint}

The primary endpoint of $\mathrm{TSS}_{2-4}$ was lower for the I-C group (5.78, $95 \%$ CI: 5.28-6.28) than for the placebo group (6.39, 95 \% CI: 5.89-6.89), but the difference was not statistically significant $(-0.61,95 \%$ CI: $-1.32-0.10$; p-value 0.0895; Table 2).

\section{Secondary and other endpoints}

For the secondary endpoints $\mathrm{SSS}_{2-4}, \mathrm{LSS}_{2-4}$, and AUC$\mathrm{TSS}_{1-10}$, there were no statistically significant differences between I-C and placebo (Table 3 ).

Mean duration of time to 'no cold' was equivalent between the groups, at 7.5 days for I-C and 7.4 days for placebo. The patients' overall assessment of efficacy was similar for I-C and placebo $(\mathrm{OR}=1.11,95 \% \mathrm{CI}$ : $0.67-$ $1.84, p=0.6954)$. For both I-C and placebo, the most frequent patient assessment was 'good' (I-C: $33.7 \%$; placebo: $36.1 \%)$ and the second most frequent assessment was 'fair' (I-C: $28.6 \%$; placebo: $33.0 \%$ ).

For TSS at each study day (Days 1-10), the mean daily TSS values were highest for both groups during the four mandatory treatment days. The TSS peaked at Day 2 in the placebo group whereas the TSS in the I-C group decreased steadily without reaching a maximum on Day 2 (Fig. 2). TSS values were lower for the I-C group than for the placebo group on Days 1-4 (mandatory treatment days); the largest differences were observed on Days 1 and 2 of treatment. After Day 4, the TSS values were lower for the placebo group than for the I-C group.

\section{Exploratory analyses of efficacy}

After unblinding, three previously unplanned analyses were performed to provide a better understanding of the primary endpoint. The data for a patient with a $\mathrm{TSS}_{2-4}$ of 16.7 (treated in the I-C group) was removed for an exploratory analysis, because his on-treatment symptom scores were well out of range when compared with the adjusted mean values of 6.39 for placebo and 5.77 for I$\mathrm{C}$ patients (Table 2), suggesting that the patient may have misunderstood the process of symptom reporting. This aberrantly high symptom score was evident over the entire treatment period of 10 days. Furthermore, no respiratory viruses were detectable in his nasopharyngeal samples. Excluding the data for this patient resulted in an adjusted mean $\mathrm{TSS}_{2-4}$ that was statistically significantly lower for the I-C group $\left(\mathrm{TSS}_{2-4}\right.$, ex 1 pt $)$ compared with the placebo group ( $p=0.0364$; Table 4$)$.

Because I-C binds to viruses and prevents their entry into cells [4, 5], an immediate effect on symptom scores could also reasonably be expected; in addition, the TSS for the placebo group was increasing from pre-treatment (baseline) to Day 2, indicating progression of the cold. Therefore, a sensitivity analysis was performed using the mean daily TSS over the mandatory treatment Days 1-4 $\left(\mathrm{TSS}_{1-4}\right)$. For the adjusted mean $\mathrm{TSS}_{1-4}$, there was a statistically significant difference in favour of I-C $(p=0.0495$; Table 4$)$. 


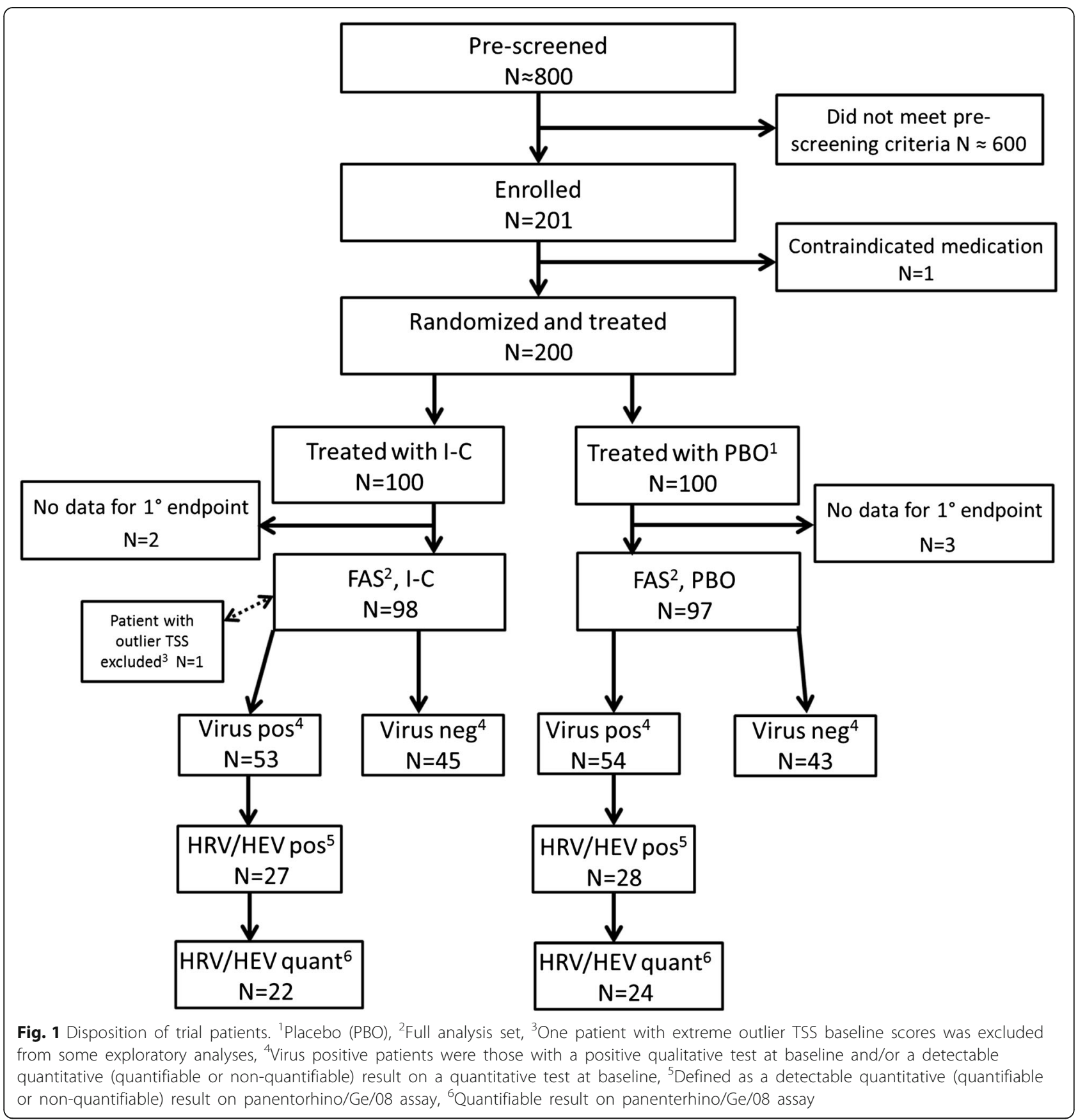

Finally, in order to adjust for the baseline severity of the cold, the $\mathrm{TSS}_{1-4}$ was analysed relative to baseline $\left(\mathrm{TSS}_{1-4, \text { rel }}\right)$, yielding a statistically significant difference in favour of I-C ( $p=0.0421$; Table 4$)$.

An exploratory analysis in the subset of 107 patients who tested positive for any virus at baseline yielded a trend in favor of I-C; average adjusted $\mathrm{TSS}_{2-4}$ scores were 0.80 points lower for the I-C group compared with placebo ( $p=0.0986$; Table 4$)$. In the subset of 55 patients who were positive for rhinovirus/enterovirus at baseline, average adjusted $\mathrm{TSS}_{2-4}$ values were 0.36 points lower than placebo $(p=0.5820$; Table 4$)$.

\section{Virologic analyses}

Including data from both qualitative and quantitative assays, the proportion of patients testing positive for virus at baseline was $54.1 \%$ (53 of 98 patients) in the I-C group and $55.7 \%$ (54 of 97 patients) in the placebo group (Table 5). In qualitative assays, the most frequent viruses identified were picornaviruses (I-C: $26.5 \%$ of 
Table 1 Demographic characteristics - treated set

\begin{tabular}{llll}
\hline & I-C & Placebo & Total \\
\hline Number of patients, N (\%) & $100(100.0)$ & $100(100.0)$ & $200(100.0)$ \\
Sex, N (\%) & & & \\
$\quad$ Male & $40(40.0)$ & $38(38.0)$ & $78(39.0)$ \\
$\quad$ Female & $60(60.0)$ & $62(62.0)$ & $122(61.0)$ \\
Race, N (\%) & & & \\
Asian & $8(8.0)$ & $2(2.0)$ & $10(5.0)$ \\
Black or African American & $2(2.0)$ & $1(1.0)$ & $3(1.5)$ \\
Hawaiian or Pacific Islander & 0 & $1(1.0)$ & $1(0.5)$ \\
$\quad$ White & $90(90.0)$ & $96(96.0)$ & $186(93.0)$ \\
Age, mean (SD) [years] & $20.01(2.37)$ & $19.93(1.90)$ & $19.97(2.14)$ \\
BMl, mean (SD) [kg/m²] & $23.98(3.67)$ & $23.66(3.89)$ & $23.82(3.78)$ \\
Smoking history, N (\%) & & & \\
Never smoked & $80(80.0)$ & $83(83.0)$ & $163(81.5)$ \\
Ex-smoker & $7(7.0)$ & $4(4.0)$ & $11(5.5)$ \\
Current smoker & $13(13.0)$ & $13(13.0)$ & $26(13.0)$ \\
Alcohol history, N (\%) & & & $9(4.5)$ \\
Does not drink & $5(5.0)$ & $4(4.0)$ & $191(95.5)$ \\
No significant drinking & $95(95.0)$ & $96(96.0)$ & \\
\hline BMl body mass index, SD standard deviation & &
\end{tabular}

$B M I$ body mass index, $S D$ standard deviation

patients, placebo: $26.8 \%$ ) and coronaviruses (I-C: $22.4 \%$, placebo: $19.6 \%$; Table 5). Between baseline and Day 3 or 4,7 of the 53 virus-positive patients in the I-C group converted to virus-negative (13.2\%), as did 8 of 54 patients in the placebo group (14.8\%). Conversely, 4 of the 44 patients in the I-C group (9.1\%) and 5 of the 43 patients in the placebo group (11.6\%) who were virus-negative at baseline converted to virus-positive by Day 3 or 4 .

As a post-hoc analysis, the quantitative data for viral types identified in at least 2 patients in each treatment

Table 2 Total symptom scores over Days 2 to $4\left(\mathrm{TSS}_{2-4}\right)$, ANCOVA-adjusted means, FAS

\begin{tabular}{|c|c|c|c|c|c|}
\hline & \multirow[b]{2}{*}{$N$} & \multirow[b]{2}{*}{$\begin{array}{l}\text { Adjusted }^{\mathrm{a}} \\
\text { mean (SE) }\end{array}$} & \multicolumn{3}{|l|}{ I-C vs. placebo } \\
\hline & & & $\begin{array}{l}\text { Adjusted }^{a} \text { mean } \\
\text { (SE) difference }\end{array}$ & $95 \% \mathrm{Cl}$ & $p$-value* \\
\hline \multicolumn{6}{|l|}{$\mathrm{TSS}_{0}{ }^{\mathrm{C}}$} \\
\hline $\mathrm{I}-\mathrm{C}$ & 98 & $6.75(0.17)$ & & & \\
\hline Placebo & 97 & $6.79(0.18)$ & & & \\
\hline \multicolumn{6}{|l|}{$\mathrm{TSS}_{2-4}$} \\
\hline I-C & 98 & $5.78(0.25)$ & $-0.61(0.36)$ & $(-1.32,0.10)$ & 0.0895 \\
\hline Placebo & 97 & $6.39(0.25)$ & & & \\
\hline
\end{tabular}

$\mathrm{Cl}$ confidence interval, SE standard error

*For ANCOVA-adjusted comparison of placebo with I-C

${ }^{a}$ The means were adjusted for $\mathrm{TSS}_{0}$

${ }^{\mathrm{b}} \mathrm{A}$ negative treatment difference favored I-C

${ }^{\mathrm{C}} \mathrm{TSS}_{0}$ values are unadjusted means
Table 3 Secondary symptom score endpoints $\mathrm{SSS}_{2-4}, \mathrm{LSS}_{2-4}$, and AUC-TSS 1-10, ANCOVA-adjusted means, FAS

\begin{tabular}{|c|c|c|c|c|c|}
\hline & \multirow[b]{2}{*}{$N$} & \multirow[b]{2}{*}{$\begin{array}{l}\text { Adjusted }^{\mathrm{a}} \\
\text { mean (SE) }\end{array}$} & \multicolumn{3}{|l|}{ I-C vs. placebo } \\
\hline & & & $\begin{array}{l}\text { Adjusted }^{a} \text { mean } \\
\text { (SE) difference }\end{array}$ & $95 \% \mathrm{Cl}$ & $p$-value \\
\hline \multicolumn{6}{|l|}{$\mathrm{SSS}_{2-4}$} \\
\hline I-C & 98 & $1.12(0.10)$ & $-0.18(0.15)$ & $(-0.46,0.11)$ & 0.2310 \\
\hline Placebo & 97 & $1.30(0.10)$ & & & \\
\hline \multicolumn{6}{|l|}{$\mathrm{LSS}_{2-4}$} \\
\hline I-C & 98 & $4.66(0.22)$ & $-0.44(0.30)$ & $(-1.04,0.16)$ & 0.1465 \\
\hline Placebo & 97 & $5.10(0.22)$ & & & \\
\hline \multicolumn{6}{|l|}{$\mathrm{AUC}^{-\mathrm{TSS}_{1-10}}$} \\
\hline I-C & 98 & $41.94(2.19)$ & $0.73(3.10)$ & $(-5.39,6.85)$ & 0.8148 \\
\hline Placebo & 97 & $41.21(2.20)$ & & & \\
\hline \multicolumn{6}{|c|}{$\begin{array}{l}\mathrm{Cl} \text { confidence interval, SE standard error } \\
{ }^{*} \text { For ANCOVA-adjusted comparison of placebo with I-C } \\
{ }^{\text {a }} \text { The means were adjusted for baseline SSS, LSS, or TSS } \\
{ }^{\mathrm{b}} \mathrm{A} \text { negative treatment difference favored I-C }\end{array}$} \\
\hline
\end{tabular}

group at baseline were analysed (coronavirus NL63, coronavirus $\mathrm{OC} 43$, and quantified panenterhino/Ge/08 assay). For the viruses quantified with the panenterhino/ $\mathrm{Ge} / 08$ assay, there was a more substantial reduction in virus load in the I-C group $(90.2 \%$ compared with $72.0 \%$ for placebo), although this did not reach statistical significance (Table 6). For the coronaviruses, decreases in mean virus quantity from baseline to Visit 2 was similar between the placebo and I-C groups (reduction of $94 \%$ or greater).

\section{Safety and tolerability}

During this trial, there were no serious AEs or deaths. Twelve patients $(6.0 \%)$ reported an AE; the frequency was similar between treatment groups, at 5 of 100 patients $(5.0 \%)$ in the I-C group and 7 of 100 patients $(7.0 \%)$ in the placebo group. AEs of severe intensity were reported for 3 of 100 patients $(3.0 \%)$ in the I-C group (one patient with both headache and migraine, one patient with toothache, and one patient with malaise) and for 5 of 100 patients $(5.0 \%)$ in the placebo group (one patient each with headache, migraine, nausea, sore throat, and fatigue). One patient in the placebo group discontinued treatment due to an $\mathrm{AE}$ (moderate epistaxis). The patients' assessment of tolerability was similar between treatment groups, with the majority rating the tolerability of the nasal spray as excellent, very good, or good (I-C: 87 patients, $88.8 \%$; placebo: 91 patients, $93.8 \%$ ). Only 1 patient in the I-C group (1.0\%) and 2 patients in the placebo group $(2.0 \%)$ assessed the tolerability of treatment as poor. Both treatments were therefore considered to be safe and well-tolerated. 


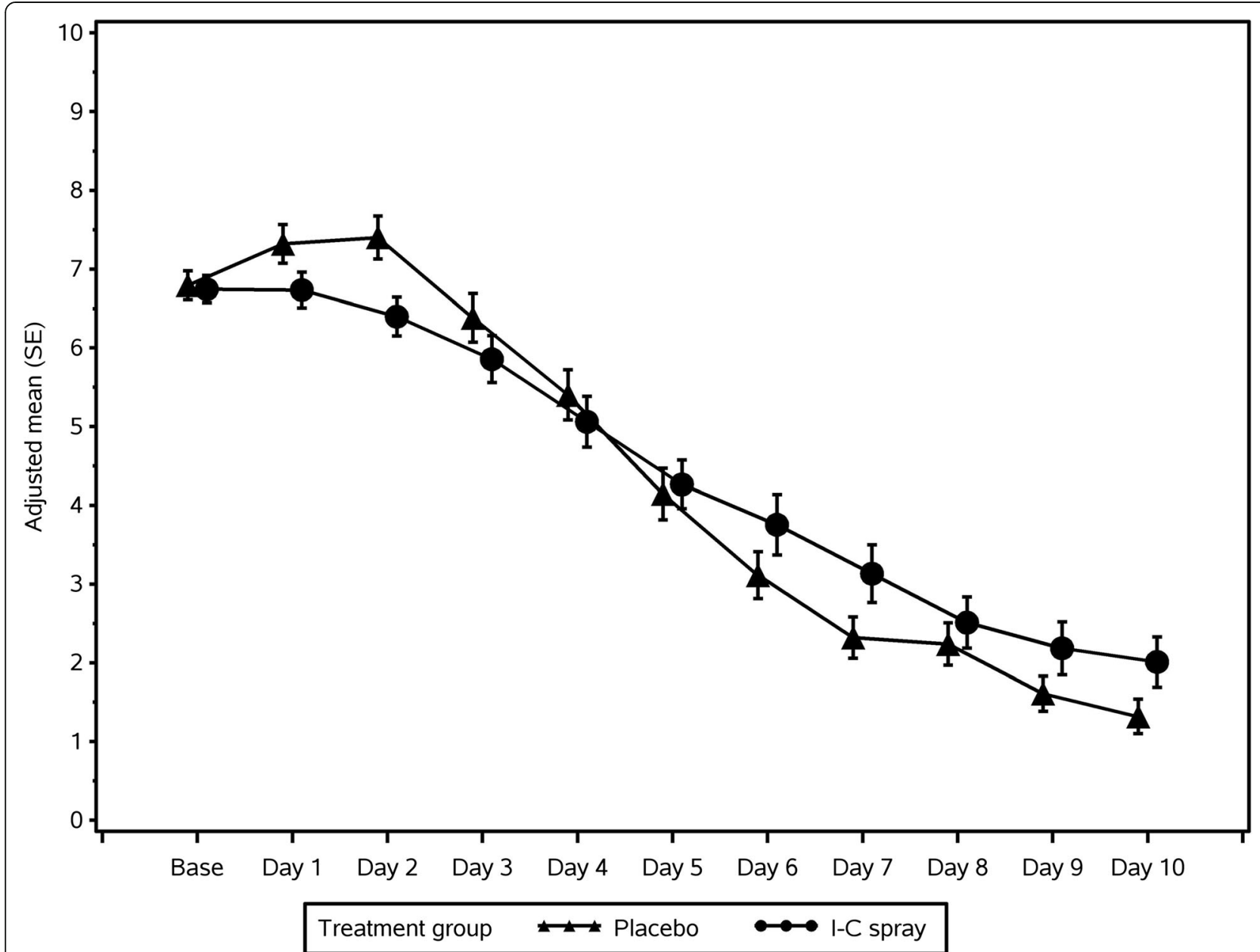

Fig. 2 Adjusted mean TSS values with standard error for each day of the trial (data at baseline are displayed as unadjusted mean values)

\section{Discussion}

This randomized, double-blind, and placebo-controlled ICICC trial was conducted to further evaluate the safety and efficacy of I-C in the treatment of patients with early common cold symptoms, and was conducted at one study site, primarily among university students who were in apparent good health except for early common cold symptoms. The analyses from the ICICC trial support the findings of three previous randomized clinical trials, which examined I-C nasal spray in adults [5, 7] and children [6] with acute common cold symptoms: in 35 adult patients, administration of I-C nasal spray significantly reduced cold symptoms and the viral concentration in nasal lavages [5]; in 203 adult patients, cold duration was significantly shorter in virus-positive patients treated with I-C, viral load was significantly reduced, and there was a significantly faster reduction of common cold symptoms after the first 3 days of treatment [7]; and in 153 children, although there was no significant difference between the I-C group and the placebo group for mean total symptom score, exploratory analyses indicated a positive effect on symptoms in patients in whom less co-medication or no co-medication was used [6]. In the ICICC trial, although the primary endpoint $\mathrm{TSS}_{2-4}$ did not demonstrate a statistically significant difference between $\mathrm{I}-\mathrm{C}$ and placebo, there was a clear trend towards benefit of I-C treatment.

Consistent trends in favor of I-C for secondary endpoints and statistically significant outcomes for exploratory analyses were also observed for the ICICC trial. The finding that nominally greater treatment effects were seen in the ICICC trial among patients who were viruspositive, is supported by a pooled analysis by Koenighofer [14], which combined the Fazekas and Ludwig trials $[6,7]$. The pooled analysis examined only those patients who were virus-positive, and showed that nominal response rates were higher in virus-positive patients than in all participants of the Ludwig and Fazekas trials.

There may be several reasons for the unexpectedly low power of the trial, which resulted in trends rather than statistically significant outcomes for the primary endpoint and HRV/HEV viral load reductions. First, the proportion of patients for whom a respiratory virus could be detected 
Table 4 Exploratory analyses of total symptom scores, ANCOVA-adjusted means

\begin{tabular}{|c|c|c|c|c|c|}
\hline & \multirow[b]{2}{*}{$N$} & \multirow[b]{2}{*}{ Adjusted $^{a}$ mean (SE) } & \multicolumn{3}{|l|}{ I-C vs. placebo } \\
\hline & & & Adjusted $^{a}$ mean (SE) difference ${ }^{b}$ & $95 \% \mathrm{Cl}$ & $p$-value* \\
\hline \multicolumn{6}{|c|}{$\mathrm{TSS}_{2-4, \text { ex } 1 \mathrm{pt}}$} \\
\hline $\mathrm{I}-\mathrm{C}$ & 97 & $5.67(0.24)$ & $-0.72(0.34)$ & $(-1.40,-0.05)$ & 0.0364 \\
\hline Placebo & 97 & $6.39(0.24)$ & & & \\
\hline \multicolumn{6}{|l|}{$\mathrm{TSS}_{1-4}$} \\
\hline $\mathrm{I}-\mathrm{C}$ & 98 & $6.02(0.21)$ & $-0.60(0.30)$ & $(-1.19,-0.00)$ & 0.0495 \\
\hline Placebo & 97 & $6.62(0.21)$ & & & \\
\hline \multicolumn{6}{|l|}{$\mathrm{TSS}_{1-4, \text { rel }}$} \\
\hline $\mathrm{I}-\mathrm{C}$ & 98 & $-0.08(0.05)$ & $-0.13(0.06)$ & $(-0.25,-0.01)$ & 0.0421 \\
\hline Placebo & 97 & $0.05(0.05)$ & & & \\
\hline \multicolumn{6}{|c|}{ Subset analyses } \\
\hline \multicolumn{6}{|c|}{$\mathrm{TSS}_{2-4}$, virus positive patients } \\
\hline $\mathrm{I}-\mathrm{C}$ & 53 & $5.87(0.34)$ & $-0.80(0.48)$ & $(-1.75,0.15)$ & 0.0986 \\
\hline Placebo & 54 & $6.67(0.34)$ & & & \\
\hline \multicolumn{6}{|c|}{$\mathrm{TSS}_{2-4}, \mathrm{HRV} / \mathrm{HEV}$ positive patients } \\
\hline $\mathrm{I}-\mathrm{C}$ & 27 & $6.08(0.46)$ & $-0.36(0.65)$ & $(-1.66,0.94)$ & 0.5820 \\
\hline Placebo & 28 & $6.44(0.46)$ & & & \\
\hline
\end{tabular}

Cl confidence interval

*For ANCOVA-adjusted comparison of placebo with I-C

aThe means were adjusted for baseline TSS

${ }^{\mathrm{b}} \mathrm{A}$ negative treatment difference favours $\mathrm{I}-\mathrm{C}$

was smaller than anticipated; respiratory viruses (all groups/types analysed) were found at baseline in only $54.9 \%$ of patients $(54.1 \%$ in the I-C group and $55.7 \%$ in the placebo group). Efforts were made to recruit patients with the earliest onset of a common cold. Thus, selfreported cold symptoms were used for patient inclusion criteria, without the requirement for demonstration of virus positivity to enter treatment. Since the early symptoms of common colds may be rather non-specific for respiratory virus infection, these symptoms may present in other conditions such as vasomotor rhinitis, in which certain nonspecific stimuli including changes in environment (temperature, humidity, or barometric pressure), airborne irritants (odors or fumes), dietary factors (spicy food or alcohol) or emotional factors trigger nasal symptoms. Patients inadvertently included with non-infectious conditions would not be expected to respond clinically to antiviral therapy, and therefore are likely to cause an under-estimation of any true antiviral treatment effect. Consistent with this, the ICICC trial demonstrated that among those patients who had quantifiable virus at baseline, the I-C patients had a greater decrease in viral load than did placebo patients, suggesting an antiviral effect of I-C. In addition, $\mathrm{TSS}_{2-4}$ scores in the patients who were virus-positive at baseline showed strong trend in favor of I-C. However, since this was a subset of the total enrolled population, the virus-positive subset was underpowered for the $\mathrm{TSS}_{2-4}$ score endpoint. In particular, the percentage of patients with HRV/HEV identified at baseline was substantially smaller than anticipated: only $23.6 \%$ had quantifiable virus $(22.4 \%$ in the I-C group and $24.7 \%$ in the placebo group). Other studies using both molecular methods and viral culture have demonstrated that human rhinoviruses are the etiologic agent in $50-90 \%$ of common colds $[1,15]$. The current trial was also conducted primarily in the fall (October until February), which includes the period of greatest HRV infection incidence $[1,15]$. Because virus shedding tends to be the greatest in the first several days of infection and generally continues for up to 2 weeks, it is unlikely that the low positive rate for HRV/HEV infection was due to missing the window of HRV shedding. More likely, the current study was conducted during a season of low HRV prevalence, resulting in fewer than expected infections among trial patients. In two previous clinical trials conducted with I-C $[6,7]$, the number of patients with confirmed HRV infection was higher than in the current trial, at $46 \%$ [6] and $30 \%$ [7] (although these values are as well lower than the percentages reported for other studies [1, 15]). Therefore, the smaller-thanexpected number of patients infected with the HRV/HEV viruses likely limited the ICICC trial's ability to differentiate between the I-C and placebo treatments.

A second issue that affected the analysis of the trial was the inclusion of one patient treated in the I-C group whose TSS scores were much higher than expected and 
Table 5 Viruses identified at baseline in trial participants

\begin{tabular}{|c|c|c|}
\hline & $\mathrm{I}-\mathrm{C}$ & Placebo \\
\hline Total number of patients tested $(\mathrm{N})$ & 98 & 97 \\
\hline Number of virus positive patients ${ }^{\mathrm{a}, \mathrm{b}}(\mathrm{N}, \%)$ & $53(54.1)$ & $54(55.7)$ \\
\hline Influenza Type A & $1(1.0)$ & $2(2.1)$ \\
\hline Picornavirus (including HRV and HEV) & $30(30.6)$ & $28(29.3)$ \\
\hline Positive qualitative picornavirus assay & $26(26.5)$ & $26(26.8)$ \\
\hline Positive qualitative enterovirus assay & $2(2.0)$ & $0(0.0)$ \\
\hline Positive result ${ }^{\complement}$ on panenterhino/Ge/08 assay & $27(27.6)$ & $28(28.9)$ \\
\hline Quantifiable result on panenterhino/Ge/08 assay & $22(22.4)$ & $24(24.7)$ \\
\hline Human metapneumovirus & $0(0.0)$ & $1(1.0)$ \\
\hline Coronavirus & $23(22.4)$ & $19(19.6)$ \\
\hline Coronavirus 229E & $1(1.0)$ & $2(2.0)$ \\
\hline Coronavirus HKU1 & $1(1.0)$ & $2(2.1)$ \\
\hline Coronavirus NL63 & $9(9.2)$ & $4(4.1)$ \\
\hline Coronavirus OC43 & $12(12.2)$ & $11(11.3)$ \\
\hline Adenovirus & $0(0.0)$ & $2(2.1)$ \\
\hline Respiratory syncytial virus & $0(0.0)$ & $1(1.0)$ \\
\hline Parainfluenza & $5(5.1)$ & $5(5.2)$ \\
\hline Type 1 & $3(3.1)$ & $0(0.0)$ \\
\hline Type 2 & $1(1.0)$ & $1(1.0)$ \\
\hline Type 4 & $1(1.0)$ & $4(4.1)$ \\
\hline
\end{tabular}

Categories are not exclusive; patients may appear in more than one category a Eight patients were positive for 2 different viruses

${ }^{b}$ No patient tested positive for influenza B, human bocavirus, human paraecho virus, or parainfluenza 3

'Detectable (quantifiable or non-quantifiable) result at baseline were much different than those of other trial participants. This patient reported exceptionally high symptom scores over the entire treatment period of 10 days; his AUC$\mathrm{TSS}_{1-10}$ was more than 3-fold higher than the overall population mean value. Interestingly, no virus was detected from this patient either at baseline or in a follow-up sample. While there is no ready explanation for these aberrant values, it is possible that this patient misinterpreted the symptom scoring methodology. When the primary endpoint $\mathrm{TSS}_{2-4}$ was re-evaluated excluding the data from this patient, the difference between the I-C and placebo patients became statistically significant.

Two other exploratory analyses further support the conclusion that I-C was efficacious. Because I-C may have had an early effect on symptoms, the assessment of on-treatment symptom scores was broadened to include Day 1. The comparison of $\mathrm{TSS}_{1-4}$ between I-C and placebo groups demonstrated a statistically significant effect of I-C, and a statistically significant change in $\mathrm{TSS}_{1-4}$ relative to baseline. Two additional exploratory analyses, although performed on very small samples, did not reach statistical significance, but also support the assertion of I-C efficacy: $\mathrm{TSS}_{2-4}$ scores in PCR-confirmed respiratory virus-positive patients were on average 0.8 points lower in the I-C group compared with placebo, and $\mathrm{TSS}_{2-4}$ scores in patients with PCR-confirmed rhinovirus infection were also on average 0.4 points lower in the I-C group.

The day-by-day TSS scores indicated an advantage for I-C on Days 1 and 2, which appeared to be lost by Days 4-5. It is not clear why the apparent TSS advantage disappeared on the later treatment days; however, the placebo was a saline nasal spray, which may have had

Table 6 Change in panenterhino/Ge/08 assay viral load in nasal secretions - patients with quantifiable baseline viral load, ANCOVAadjusted means

\begin{tabular}{|c|c|c|c|c|c|}
\hline & \multirow[b]{2}{*}{$\mathrm{N}$} & \multirow[b]{2}{*}{ Adjusted $^{\mathrm{a}}$ mean (SE) } & \multicolumn{3}{|l|}{ I-C vs. placebo } \\
\hline & & & Adjusted $^{a}$ mean (SE) difference ${ }^{b}$ & $95 \% \mathrm{Cl}$ & $p$-value* \\
\hline \multicolumn{6}{|c|}{ Baseline [log (copies/mL) $]^{c}$} \\
\hline $1-C$ & 22 & $5.840(0.200)$ & & & \\
\hline Placebo & 24 & $6.139(0.201)$ & & & \\
\hline \multicolumn{6}{|c|}{ Change from baseline, [log (copies/mL)] } \\
\hline $\mathrm{I}-\mathrm{C}$ & 22 & $-1.007(0.191)$ & $-0.453(0.266)$ & $-0.990,0.084$ & 0.0958 \\
\hline Placebo & 24 & $-0.554(0.183)$ & & & \\
\hline \multicolumn{6}{|c|}{ Reduction from baseline $(\%)^{c, d}$} \\
\hline $1-C$ & 22 & 90.2 & & & \\
\hline Placebo & 24 & 72.0 & & & \\
\hline
\end{tabular}

Cl confidence interval, SE standard error

*For ANCOVA-adjusted comparison of placebo with I-C

${ }^{a}$ The means were adjusted for baseline viral load

${ }^{\mathrm{b}} \mathrm{A}$ negative treatment difference favored $\mathrm{I}-\mathrm{C}$

'Baseline values are unadjusted means

${ }^{\mathrm{d} C a l c u l a t e d}$ according to Eccles et al. [5] 
some clinical benefit from saline irrigation of the nasal passages [16-18].

The I-C spray is registered and marketed as medical device in several European countries and is intended to be used in patients with early cold symptoms. Data from several experiments suggest that the mechanism of action may be prevention of binding to attachment sites and/or interference of viral entry. First, in vitro studies have demonstrated activity against a variety of viruses - various strains of HRV and influenza and herpesvirus, denguevirus, and papillomavirus [3, 4, 19-21]. This non-specificity suggests the absence of a specific direct-acting antiviral (DAA). Secondly, the observed antiviral effects occurred early in the infection cycle, most likely during the stages of attachment and/or entry, and the antiviral effect tended not to be I-C concentration-dependent. It is, therefore, unlikely that carrageenan's effect is that of a DAA targeted to the metabolic or reproductive mechanisms. Since a variety of viruses cause the common cold and since the virus infection cycles typically involve binding and entry with nasal mucosal cells, the antiviral properties of iota carrageenan represents an attractive and promising option for treatment.

Published clinical evidence concerning the frequencies of detectable viruses varies but with modern techniques, viruses (particularly rhinoviruses) have been found in up to $90 \%$ of patients suffering from cold symptoms. It can therefore be expected that in real-life conditions patients/consumers who empirically choose to use the I-C spray have a relatively high likelihood of being infected with rhinovirus or one of a variety of respiratory viruses that manifest with the common cold symptoms. The uncertainty of true infection in such a setting is counterbalanced by the very low intolerance and toxicity rates of the I-C spray and its relative inexpensiveness, thus giving favorable potential benefit-risk and benefit-cost ratios to the empiric use of the I-C spray.

In clinical trials of cough \& cold, a substantial proportion of studies demonstrate trends but may not reach statistically significant efficacy outcomes. This observation may be due to the large variability in the disease severity between native infection and patients recruited in the early stages of a cold. When a controlled clinical trial tries to recruit patients at the earliest stages of a common cold - when symptoms are still emerging and are mild - patients may incorrectly believe they are coming down with a cold, prior to full blown cold symptoms. The relatively low frequency of rhinovirus-positive patients in the ICICC study demonstrates that there is often a trade-off when this standard design is used for cold studies. The peculiarities of the outcome of the ICICC study are also useful in that they may trigger a discussion among the scientific community about more suitable study designs to investigate common cold treatments.

Furthermore, the results of the ICICC study are in line with many other common cold studies which showed relatively small effects of treatment on symptom severity or duration. One example is the study by Barrett et al. on Echinacea which showed as well trends in the direction of benefit, amounting to an average half day reduction in the duration of a week-long cold, or an approximate $10 \%$ reduction in overall severity. The authors concluded that "while these results do not allow us to reject the null hypothesis and confidently claim evidence-of-benefit, data are also insufficient to exclude the possibility of a clinically significant effect" [22].

Although not addressed in the ICICC trial, I-C's antiviral properties lead to speculation that its use might decrease cold transmission to patients' family members and other social contacts.

\section{Conclusions}

Although the ICICC study failed to reach statistically significant outcomes for the primary and secondary endpoints, strong trends favouring treatment effect of I-C were observed. The primary and secondary endpoints consistently tended to be improved in the I-C spray group compared to the placebo group, with clinically relevant effect sizes, even though the population included patients with low baseline scores. Several rational and important exploratory analyses led to statistically significant and clinically relevant outcome differences. Unfortunately, the relatively low numbers of subjects with demonstrated viral infection limited study conclusions. Nonetheless, the outcome of the ICICC study is supported by previous evidence of I-C's in vitro antiviral activity and symptom and viral reduction in prior cold studies. The ICICC trial's outcome supports I-C spray as representing a potentially useful treatment option for the common cold. Trial results show it to be safe, well-tolerated, and minimally invasive, as well as suggesting efficacy.

\section{Competing interests}

The study was sponsored by Boehringer Ingelheim Pharma GmbH \& Co KG, Ingelheim am Rhein, Germany.

Patrick Robinson, Matthias Trampisch and Stephan Koelsch are employees of the sponsor.

Ronald Eccles was remunerated by the sponsor for his services as principal and coordinating investigator. Birgit Winther and Sebastian Johnston were reimbursed for their scientific advice for this study on a contractual basis by the sponsor.

\section{Authors' contributions}

Conception and design of the study was developed by the sponsor under the responsibility of SK. Principal investigator was RE. Statistical design and analysis was the responsibility of MT. Overall evaluation, interpretation of the data and preparation of the manuscript was the joint effort of all listed authors as coordinated by SK. All authors reviewed the manuscript critically and approved the final version to be published. At no time the sponsor took undue influence on the data presented here and the conclusions drawn from them. 


\section{Acknowledgements}

We acknowledge Dr. Jill Holbrook who provided medical writing services on behalf of BI Pharma GmbH \& Co. KG. We thank the staff at the Common Cold Centre: Dr. Moutaz Jawad, Dr. Eugene Wong, Miss Suhair Jawad, Mr. Ben Pope and Miss Edyta Zwolska. We acknowledge Prof Laurent Kaiser, University Hospitals of Geneva, Division of Infectious Diseases, Division of Laboratory Medicine - Laboratory of Virology, Geneva, Switzerland and his staff, Dr. Caroline Tapparel, Aline Mamin and Yves Thomas, for performing the virus analysis.

\section{Author details}

${ }^{1}$ Common Cold Centre, Cardiff School of Biosciences, Cardiff University, Cardiff, UK. ${ }^{2}$ Respiratory Disease Study Center, University of Virginia, Charlottesville, VA, USA. ${ }^{3}$ Airway Disease Infection Section \& MRC \& Asthma UK Centre in Allergic Mechanisms of Asthma, National Heart and Lung Institute, Imperial College, London, UK. ${ }^{4}$ Boehringer Ingelheim Pharmaceuticals Inc., Therapeutic Area Virology, Ridgefield, CT, USA. ${ }^{5}$ Boehringer Ingelheim Pharma GmbH \& Co. KG, Biometrics \& Data Management, Ingelheim/Rhein 55216, Germany. ${ }^{6}$ Boehringer Ingelheim Pharma GmbH \& Co. KG, CHC Development, Medicine \& Regulatory Affairs, Ingelheim/Rhein 55216, Germany.

Received: 17 June 2015 Accepted: 18 September 2015

\section{Published online: 05 October 2015}

\section{References}

1. Mäkelä MJ, Puhakka T, Ruuskanen O, Leinonen M, Saikku P, Kimpimäki M, et al. Viruses and bacteria in the etiology of the common cold. J Clin Microbiol. 1998;36:539-42.

2. Fendrick MA, Monto AS, Nightengale B, Sarnes M. The Economic Burden of Non-Influenza-Related Viral Respiratory Tract Infection in the United States. Arch Intern Med. 2003;163:487-94. http://dx.doi.org/10.1001/archinte.163.4.487.

3. Leibbrandt A, König-Schuster M, Weinmüllner R, Kalthoff D, Pflugfelder B, Graf $P$, et al. lota-Carrageenan is a potent inhibitor of influenza A virus infection. PLoS One. 2010;5:e14320. http://dx.doi.org/10.1371/ journal.pone.0014320.

4. Grassauer A, Weinmuellner R, Meier C, Pretsch A, Prieschl-Grassauer E, Unger $\mathrm{H}$. lota-carrageenan is a potent inhibitor of rhinovirus infection. Virol $\mathrm{J}$. 2008:5:107. http://dx.doi.org/10.1186/1743-422x-5-107.

5. Eccles R, Meier C, Jawad M, Weinmueller R, Grassauer A, Prieschl-Grassauer E. Efficacy and safety of an antiviral lota-Carrageenan nasal spray: a randomized, double-blind, placebo-controlled exploratory study in volunteers with early symptoms of the common cold. Respir Res (Lond). 2010;11:108. http://dx.doi.org/10.1186/1465-9921-11-108

6. Fazekas T, Eickhoff P, Pruckner N, Vollnhofer G, Fischmeister G, Diakos C, et al. Lessons learned from a double-blind randomised placebo-controlled study with an iota-carrageenan nasal spray as medical device in children with acute symptoms of common cold. BMC Complement Altern Med. 2012;12:147. http://dx.doi.org/10.1186/1472-6882-12-147.

7. Ludwig M, Enzenhofer E, Schneider S, Rauch M, Bodenteich A, Neumann $\mathrm{K}$, et al. Efficacy of a carrageenan nasal spray in patients with common cold: a randomized controlled trial. Respir Res (Lond). 2013;14:124. http://dx.doi.org/10.1186/1465-9921-14-124.

8. Jackson GG, Dowling HF, Spiesman IG, Boand AV. Transmission of the common cold to volunteers under controlled conditions. Intern Med. 1958;101:267-78

9. Allen EK, Pitkaranta A, Maki M, Hendley JO, Laakso S, Sale MM, Winther B. Bacteria in the nose of young adults during wellness and rhinovirus coldsdetection by cultures and microarray methods in 100 nasal lavage specimens. Int Forum Allergy Rhinol. 2013;9:731-39.

10. Tapparel C, Cordey S, Van Belle S, Turin L, Lee WM, Regamey N, et al. New Molecular Detection Tools Adapted to Emerging Rhinoviruses and Enteroviruses. J Clin Microbiol. 2009;47:1742. http://dx.doi.org/10.1128/JCM.02339-08.

11. Schibler M, Yerly S, Vieille G, Docquier M, Turin L, Kaiser L, Tapparel C. Critical Analysis of Rhinovirus RNA Load Quantification by Real-Time Reverse Transcription-PCR. J Clin Microbiol. 2012;50:2868. http://dx.doi.org/10.1128/JCM.06752-11.

12. Garbino J, Crespo S, Aubert JD, Rochat T, Ninet B, Deffernez C, et al. A prospective hospital-based study of the clinical impact of non-severe acute respiratory syndrome (non-SARS)-related human coronavirus infection. Clin Infect Dis. 2006;43:1009.
13. Garbino J, Gerbase MW, Wunderli W, Deffernez C, Thomas Y, Rochat T, et al. Lower respiratory viral illnesses: improved diagnosis by molecular methods and clinical impact. Am J Respir Crit Care Med. 2004;170:1197.

14. Koenighofer M, Lion T, Bodenteich A, Prieschl-Grassauer E, Grassauer A, Unger H, Mueller CA, Fazekas T. Carrageenan nasal spray in virus confirmed common cold: individual patient data analysis of two randomized controlled trials. Multidisciplinary Respir Med 2014, 9:57

15. Arruda E, Pitkaranta A, Witek TJ, Doyle CA, Hayden FG. Frequency and natural history of rhinovirus infections in adults during autumn. J Clin Microbiol. 1997;35:2864-8.

16. Rabago D, Pasic T, Zgierska A, Mundt M, Barrett B, Maberry R. The efficacy of hypertonic saline nasal irrigation for chronic sinonasal symptoms. Otolaryngol Head Neck Surg. 2005;133:3-8.

17. Rabago D, Zgierska A, Mundt M, Barrett B, Bobula J, Maberry R. Efficacy of daily hypertonic saline nasal irrigation among patients with sinusitis: a randomized controlled trial. J Fam Pract. 2002;51:1049-55.

18. Kassel JC, King D, Spurling GK. Saline nasal irrigation for acute upper respiratory tract infections. Cochrane Database Syst Rev. 2010;3:CD006821. doi:10.1002/14651858.CD006821.pub2

19. Carlucci MJ, Ciancia M, Matulewicz MC, Cerezo AS, Damonte EB. Antiherpetic activity and mode of action of natural carrageenans of diverse structural types. Antiviral Res. 1999;43:93-102.

20. Talarico LB, Damonte EB. Interference in dengue virus adsorption and uncoating by carrageenans. Virology. 2007;363:473-85.

21. Buck CB, Thompson CD, Roberts JN, Muller M, Lowy DR, et al. Carrageenan is a potent inhibitor of papillomavirus infection. PLoS Pathog. 2006;2:e69-ff.

22. Barrett B, Brown R, Rakel D, Mundt M, Bone K, Barlow S, et al. Echinacea for treating the common cold: a randomized controlled trial. Ann Intern Med. 2010;153(12):769-77.

\section{Submit your next manuscript to BioMed Central and take full advantage of:}

- Convenient online submission

- Thorough peer review

- No space constraints or color figure charges

- Immediate publication on acceptance

- Inclusion in PubMed, CAS, Scopus and Google Scholar

- Research which is freely available for redistribution

Submit your manuscript at www.biomedcentral.com/submit
C Biomed Central 